\title{
MOA-II Microlensing survey
}

\author{
Takahiro Sumi ${ }^{1}$ \\ Nagoya University, Furou-cho, Chikusa-ku, Nagoya, Aichi 464-8601, Japan \\ E-mail: sumi@stelab.nagoya-u.ac.jp
}

Abe, F; Bennett, P. D;Bond, I. A.; Fukui, A;Furusawa, K; Hearnshaw, J. B.; Itow, Y; Kilmartin, P. M.; Koki, K;Masuda, K.; Matsubara, Y.; Miyake, N; Muraki, Y.; Nagaya, M;Okumura,M; Ohnishi,K; Rattenbury, N. J.; Saitou,T; Sako, T.; Sullivan, D. J.; Tristram, P.; Wood, J. N.; Yock, P. C. M.

\begin{abstract}
Microlensing Observations in Astrophysics (MOA) has been caring out a microlensing survey at Mt. John observatory in New Zealand since 1995. We observe several tens of millions of stars in the Galactic Bulge (GB) and the Magellanic Clouds (LMC and SMC) for studying the exoplanets and the Galactic dark mater. The first phase of MOA, MOA-I, which used to use $60 \mathrm{~cm}$ telescope, found one microlensing event toward the LMC. MOA-I also contributed to the discovery of two exoplanets including the first exoplant via microlensing. The second phase of MOA, MOA-II, is currently using a $1.8 \mathrm{~m}$ dedicated telescope with 2.2 square degree field of view (FOV). Thanks to this wide FOV, we are observing 24 square degrees field every 2 hours and 7 square degrees every $15 \mathrm{~min}$ toward the LMC. This high frequent sampling is important to detect anomaly, such as parallax, finite source effect and binary events, in which we can get additional information about where the lens is. Furthermore, it is helpful to distinguish from the background Supper Novae that were the major contamination in the previous large survey. MOA-II detected $\sim 500$ microlensing events towards the GB and issued alerts to other groups in real-time to encourage follow-up observations in 2007 season. We are observing the GB fields very frequently, 50 square degree every an hour and 4.5 square degree every $10 \mathrm{~min}$., which enable us to find short time scale planetary deviation. In 2007, we started the new system "real-time anomaly alert system" which plot new data points on the alerted microlesning events 5min. after each exposure. If the observer sees any anomalous point from the standard light curve, an additional exposure is taken immediately to confirm the anomaly. Then the anomaly alert is issued if the anomaly was confirmed. We issued the several anomaly alerts in 2007. One of them was a real planetary deviation, OGLE-2007-BLG-368 (MOA-2007-BLG-308). Our high cadence observation and "real-time anomaly alert system" is especially effective to find the planetary caustic events that we cannot predict when it will happen.
\end{abstract}

The Manchester Microlensing Conference: The 12th International Conference and ANGLES Microlensing Workshop

Manchester, $U K$

21-25 January, 2008

$1 \quad$ Speaker 